5 Swinburne A.J. Augustine ${ }^{\mathrm{a} *}$, Kaneatra J. Simmons ${ }^{\mathrm{a}}$, Tarsha N. Eason ${ }^{\mathrm{b}}$, Shannon M. Griffin ${ }^{\mathrm{a}}$, 6 Clarissa L. Curioso ${ }^{c}$, Larry J. Wymer ${ }^{\mathrm{a}}$, G. Shay Fout ${ }^{\mathrm{a}}$, Ann C. Grimm ${ }^{\mathrm{a}}$, Kevin H. Oshima ${ }^{\mathrm{a}}$, Al 7

8

9

\section{Statistical approaches to developing a multiplex immunoassay for determining human exposure to environmental pathogens}

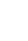

$$
\text { Dufour }^{\mathrm{a}}
$$

${ }^{\mathrm{a}}$ National Exposure Research Laboratory, U.S. Environmental Protection Agency, 26 W. Martin Luther King Drive, Cincinnati, OH 45268, USA

${ }^{\mathrm{b}}$ National Risk Management Research Laboratory, U.S. Environmental Protection Agency, 26

W. Martin Luther King Drive, Cincinnati, OH 45268, USA

${ }^{\mathrm{c}}$ Oak Ridge Institute for Science and Education, Oak Ridge, TN 4 5

* Corresponding author at: 26 W. Martin Luther King Drive, Cincinnati, OH 45268, USA.

Telephone: (513) 569-7132. E-mail address: augustine.swinburne@epa.gov (S. Augustine).

8

9

0

1

3




\section{Abstract}

25 There are numerous pathogens that can be transmitted through water. Identifying and understanding the routes and magnitude of exposure or infection to these microbial

27 contaminants are critical to assessing and mitigating risk. Conventional approaches of studying immunological responses to exposure or infection such as Enzyme-Linked Immunosorbent Assays (ELISAs) and other monoplex antibody-based immunoassays can be very costly, laborious, and consume large quantities of patient sample. A major limitation of these approaches is that they can only be used to measure one analyte at a time. Multiplex immunoassays provide the ability to study multiple pathogens simultaneously in microliter volumes of samples. However, there are several challenges that must be addressed when developing these multiplex immunoassays such as selection of specific antigens and antibodies, cross-reactivity, calibration, protein-reagent interferences, and the need for rigorous optimization of protein concentrations. In this study, a Design of Experiments

37 (DOE) approach was used to optimize reagent concentrations for coupling selected antigens to Luminex ${ }^{\mathrm{TM}} \mathrm{xMAP}$ microspheres for use in an indirect capture, multiplex immunoassay to detect human exposure or infection from pathogens that are potentially transmitted through were used to determine the mean concentrations that would be applied to the multiplex assay.

42 Cut-offs to differentiate between exposed and non-exposed individuals were determined using finite mixed modeling (FMM). The statistical approaches developed facilitated the

44 detection of Immunoglobulin $\mathrm{G}(\mathrm{IgG})$ antibodies to H. pylori, C. jejuni, T. gondii, hepatitis A 45 virus, rotavirus and noroviruses (VA387 and Norwalk strains) in fifty-four diagnostically 46 characterized plasma samples. Of the characterized samples, the detection rate was $87.5 \%$ 47 for H. pylori, and $100 \%$ for T. gondii assays and $89 \%$ for HAV. Further, the optimized 
48 multiplex assay revealed exposure/infection to several other environmental pathogens

49 previously uncharacterized in the samples.

50

51 Keywords: Multiplex immunoassay, assay optimization, Design of Experiments (DOE),

52 response surface methods (RSM), finite mixed modeling (FMM)

53

54

55

56

57

58

\section{Introduction}

Conventional approaches to studying human exposure or infection from pathogens

60

61

62

63

64

65

66

67 include epidemiological surveys that collect symptomology type data and ELISAs that detect the presence of antibodies specific to pathogens. Determination of exposure or infection from pathogens using survey-based epidemiological studies can be challenging because of difficulties enrolling participants and high cost. These observational survey-based approaches may suffer from selection bias and problems of recall (Lilienfeld 1983). Additionally, determination of the etiologic agents may be challenging because symptoms and incubation periods may not be unique to particular pathogens. Combining epidemiologic assessment with assays to measure 
immunological status can be very effective in determining which pathogens present exposure risks to a given population.

Recent technological advances have provided the ability to study multiple analytes simultaneously with very small sample volumes (Ellington et al., 2010; Fu et al., 2010; Choi et al., 2013). One such technology, the Luminex xMAP (Luminex Corp., Austin, TX) bead-based immunoassay is theoretically capable of multiplexing up to 500 unique assays in as little as $50 \mu \mathrm{l}$ of sample. This is in contrast to ELISA-type antibody-based immunoassays that can measure only one analyte at a time, are time-consuming, require larger volumes of patient sample for multiple tests, and are often costly. However, well-designed immunoassays and assay optimization tests are time consuming and costly due to the number of factors that must be managed. They require determining settings for multiple parameters and balancing sample availability with the use of expensive reagents and equipment. Hence, it is important that these assays are systematically developed to ensure they are precise and produce optimal results.

Determining key or critical assay parameters typically involve evaluating output response given changes in parameter settings one-factor-at-a-time (OFAT). In addition to being time and resource intensive, OFAT provides no means of determining or exploring important interactions between factors. As an alternative, DOE offers an efficient means of investigating the impact of changes in system parameters, exploring factor combinations and identifying parameter settings for optimizing experimental results (Montgomery 1997; Khuri and Mukhopadhyay 2010). DOE has effectively been used in assay development to provide critical insight for optimizing experimental conditions for applications to include the detection of anti-drug neutralizing antibodies in human serum; optimization of an automated digital microfluidic platform immunoassay; ligand-binding assay sensitivity; optimizing ELISAs; and measuring neutralizing 
91 antibodies against Clostridium difficile toxins (Chen et al., 2012; Choi et al., 2013; Joelsson et

92 al., 2008; Joyce and Leung 2013; Sitta Sittampalam et al., 1996; Xie et al. 2013).

The goals of the present study are to optimize reagent concentrations for detecting human

94 IgG antibodies in characterized human plasma samples and determine cut-off points for the antigens in the assay using FMM (Baughman et al., 2004). Multiplex immunoassays are not as

96 common as singleplex methods and have the additional requirement of needing multiple

97 optimizations. To handle this complexity, we employ the use of DOE for designing and optimizing an assay for simultaneously detecting antibodies to multiple pathogens in commercial samples. The optimized settings from these tests will later be used to investigate bathing related exposures or infections from waterborne pathogens.

101

102

\section{Materials and Methods}

Designing a Luminex-based, indirect capture, multiplex immunoassay requires determining the optimal concentrations for antigen $(\mathrm{Ag})$, primary antibody $\left(1^{\circ} \mathrm{Ab}\right)$, biotinylated 105 secondary antibody $\left(2^{\circ} \mathrm{Ab}\right)$, and reporters, as well as, determining the optimal $\mathrm{pH}$ for coupling of antigens to the microspheres. A pH of 5.0 was previously determined to be best for the coupling reactions (Griffin et al., 2011) and we found that $2^{\circ} \mathrm{Ab}$ concentration and streptavidinR-phycoerythrin (SAPE) (Life Technologies, Carlsbad, CA, USA) worked best as recommended by Luminex $\left(\mathrm{SAPE}\right.$ concentration $=$ biotinylated $2^{\circ} \mathrm{Ab}$ concentration $\left.\times 1.5\right)$. Preliminary experiments showed that the reagent concentrations necessary to achieve MFIs that approached 111 the upper Limit of Detection (LoD) of the Luminex instrument (up to 30,000 MFI) tended to 112 vary from antigen to antigen; accordingly, a range of concentrations was tested to optimize each 113 assay (Table 1). 
Multiple sets of xMAP carboxylated microspheres were obtained from Luminex Corp.

115 (Austin, TX, USA) at a concentration of $12.5 \times 10^{6}$ beads/ ml each. Biotin-labeled affinity

116 purified goat anti-human $\operatorname{IgG}(\lambda)$ and rabbit anti-goat $\operatorname{IgG}(\mathrm{H}+\mathrm{L})$ were obtained from $\mathrm{KPL}$,

117 (Gaithersburg, MD, USA). Biotin-labeled donkey anti-rabbit IgG $(\mathrm{H}+\mathrm{L})$ and donkey anti-guinea

118 pig IgG $(\mathrm{H}+\mathrm{L})$ were purchased from Jackson ImmunoResearch, (West Grove, PA, USA).

2.1. Two-step carbodiimide coupling of antigens to $x M A P^{\circledR}$ carboxylated beads and confirmation of antigen coupling using animal-derived antibodies:

The Luminex microspheres were activated and coupled as described in Griffin et al.,

123 (2011). Coupling confirmation was performed using serial dilutions of commercially available,

124 animal-derived primary capture antibodies specific to each antigen to ensure that the beads were coupled properly and that the dynamic range of the assay could be achieved. Briefly, a working

126 bead mixture was prepared by diluting the coupled bead stocks to a final concentration of 100

127 beads/ $\mu$ l of each unique bead set in PBS-1\% bovine serum albumin (BSA). Two-fold serial

128 dilutions of anti-species IgG primary antibody were prepared per the manufacturer's

129 recommendations. A MultiScreen BV 96 well $1.2 \mu \mathrm{m}$ filter plate (Millipore, Billerica, MA,

130 USA) was pre-wet with $100 \mu$ l of PBS-1\% BSA, excess buffer was removed with a vacuum 131 manifold and $50 \mu 1$ of the working bead mixture was added to each well. The serially diluted 132 species-specific antibody was added to the beads, the reactions were mixed gently with a multi133 channel pipettor, covered, and allowed to incubate in the dark, at room temperature for $30 \mathrm{~min}$ at $134500 \mathrm{rpm}$ on a VWR ${ }^{\mathrm{TM}}$ microplate shaker (Radnor, PA, USA).

135 The supernatant was removed by vacuum manifold, and the wells were washed twice 136 with $100 \mu \mathrm{l}$ of PBS-1\% BSA, 0.05\% Tween 20 buffer and excess buffer removed by vacuum 
manifold. The beads were gently resuspended in $50 \mu 1$ of PBS-1\% BSA buffer with a multichannel pipettor, and $50 \mu \mathrm{l}$ of a $16 \mu \mathrm{g} / \mathrm{ml}$ of biotinylated anti-species IgG secondary detection antibody was added to each well. The filter plates were covered and allowed to incubate in the dark at room temperature for 30 min on a plate shaker. After incubation, the wells were washed twice with $100 \mu 1$ of PBS- 1\% BSA, 0.05\% Tween 20 buffer and excess buffer removed by vacuum manifold. Finally, $50 \mu 1$ of $24 \mu \mathrm{g} / \mathrm{ml} \mathrm{SAPE}$ was added to each well, mixed gently with a multi-channel pipettor and incubated as above for 30 minutes and washed twice as above. Excess buffer was removed by vacuum manifold, the beads were resuspended in $100 \mu 1$ of PBS-1\% BSA, and the plate analyzed on a Luminex 100 analyzer.

\subsection{DOE optimization of assay parameters}

Design Expert 8.0.7.1 (StatEase, Inc., Minneapolis, MN, USA) was used to design singleplex experiments using antigens to $H$. pylori, C. jejuni, E. coli $\mathrm{O} 157: \mathrm{H} 7$, and $S$. typhimurium. The concentration of $\mathrm{Ag}$ coupled to the microspheres, $1^{\circ} \mathrm{Ab}, 2^{\circ} \mathrm{Ab}$ and SAPE were used as factors. Since $2^{\circ} \mathrm{Ab}$ concentration and SAPE vary together, the SAPE was used as an experimental factor but eliminated as a design factor. A full factorial design was initially used to assess the impact of factor changes on MFI response for all of the pathogens, after which, response surface methods (RSM) were employed in accordance with the optimization goal of this work. RSM uses statistical and mathematical approaches to explore the functional relationship between controllable factors and output response. They are used for modeling and analyzing problems with multiple parameters, determining factor significance, developing mathematical models and optimizing system response (Montgomery 1997). There are a number of RSM approaches to include D-optimal, central composite designs (CCD) and Box-Behnken 
160 designs (Khuri and Mukhopadhyay 2010, Montgomery 1997). Each can be employed in a

161 variety of circumstances; however, we used a D-Optimal design for our studies. A D-Optimal

162 design is a RSM developed to select design points in a way that minimizes the variance

163 associated with the estimates of the specified model coefficients (Khuri and Mukhopadhyay

164 2010).

165

166

2.3. Developing the complete multiplex based on DOE results

167

Singleplex immunoassays were performed for the following organisms: H. pylori, $C$.

168 jejuni, E. coli O157:H7, and S. typhimurium to determine the optimal parameter settings for each

169 antigen/antibody pair. After using DOE to numerically optimize the $\mathrm{Ag}, 1^{\circ}$ and $2^{\circ} \mathrm{Ab}$

170 concentrations for each organism, the results for each reagent was pooled and a mean was

171 computed from these results. The mean concentration value for each reagent was then applied to

172 hepatitis A virus, rotavirus, and T. gondii. The noroviruses had been previously optimized in

173 Griffin et al. (2011). Antigen coupling and confirmation were conducted as described in Section

1742.1 above. The complete multiplex immunoassay was performed on 96 well plates and analyzed

175 for assay sensitivity and specificity.

176 Assay specificity was evaluated by performing single detection cross-reactivity using the

177 concentration of $1^{\circ} \mathrm{Ab}$ at the highest point of the linear range of the assay. Individual $1^{\circ} \mathrm{Abs}$

178 were tested in the presence of a multiplex of antigen-coupled beads and the appropriate

179 biotinylated $2^{\circ} \mathrm{Ab}$ and SAPE. The degree of cross-reactivity (defined as the percentage of MFI

180 detected for a given antigen analyte relative to the specific MFI for a target antigen analyte) was

181 calculated for all antigens. An assay was excluded from the multiplex if the maximum reporter 
182 signal observed in coupling confirmation tests was less than 18,000 MFI and/or cross-reactivity 183 to any non-target antigen was greater than $10 \%$.

2.4. Assay validation of the optimized DOE parameters using commercially obtained diagnostically characterized human plasma samples

Serial dilutions (ranging from 1:20 to 1:6400) of characterized human plasma samples were tested using the beads that had been confirmed to be properly coupled with animal-derived anti-species antibodies to determine the dynamic ranges of those samples. A total of 54 commercially obtained plasma samples were analyzed in this study (44 positives and 10 negatives). 33 plasma samples were purchased from Access Biologicals (Vista, CA, USA) and

19221 samples were obtained from SeraCare Life Sciences (Milford, MA, USA). These plasma 193 samples were from patients diagnostically characterized with $H$. pylori and $T$. gondii $((\mathrm{n}=16(5$ 194 positives and 3 negatives each)), HAV (( $\mathrm{n}=17$ (14 positives and 3 negatives $))$, L. pneumophila $195(\mathrm{n}=10)$ and S. typhimurium $((\mathrm{n}=11$ (10 positives and 1 negative $))$ infections. The L. pneumophila 196 plasma was donated by SeraCare but the vendor was not confident about positivity. With the 197 exception of the L. pneumophila samples, the characterization of the remaining 44 plasma 198 samples was confirmed by the suppliers: Access Biologicals, using the ADVIA Centaur kits 199 (Siemens Healthcare Diagnostics Inc., Deerfield, IL, USA) and SeraCare Life Sciences using 200 Calbiotech EIA (Calbiotech, Spring Valley, CA, USA) for the target organisms. The plasma 201 samples were first tested by the Luminex platform using the optimized DOE reagent 202 concentrations to confirm that they were either positive or negative. Once the results were 203 validated in singleplex by Luminex, the characterized samples were exposed to a multiplex of 204 the antigen-coupled bead sets. 
2.5. Assay controls, cross-reactivity and signal to noise ratio ( $S / N)$

Two assay controls were utilized to ensure proper performance of the multiplex

208 immunoassay. First, one set of beads was used as a control for nonspecific binding of antibodies

209 to the beads. These beads were treated exactly like the actual bead sets used in the analysis

210 except that they were not coupled to any antigen before being exposed to the antibodies. The

211 second control consisted of antigen-coupled beads but the antibodies were replaced with $1 \%$

212 PBS-BSA. The MFI values from the background controls (no antigen microspheres and antigen-

213 coupled beads with no primary antibody) were used to determine non-specific binding and the

214 MFI values were subtracted from each sample result.

215 Several cross-reactivity assays were performed with the animal-derived antibodies

216 including monoplex (one antigen with its specific antibody), duplex (one antigen and its specific

217 antibody) and multiplex (multiple antigens, one antibody and controls). Since it was not possible

218 to obtain human-derived positive and negative controls for each antigen, a determination was

219 made to remove antigen/antibody pairs that demonstrated levels of cross-reactivity $>10 \%$ using

220 the animal-derived primary antibodies. As a surrogate to measure assay sensitivity, a signal to

221 noise ratio $(\mathrm{S} / \mathrm{N})$ was calculated by dividing the specific antigen signals by the MFI of the

222 uncoupled control beads.

223

224 2.6. Establishing cutoffs to distinguish exposure status: exposed vs. unexposed

225 Given the lack of information regarding the true status of exposure/infection for the

226 uncharacterized antigens in the multiplex, we used finite mixed models (FMM) (Titterington et

227 al., 1985; McLachlan and Peel, 2000) to predict exposure sub-group distributions based on 
228 MFIs. Parameters for respective sub-group membership distributions were estimated via

229 maximum likelihood for a family of models using the expectation-maximization technique due to

230 the incomplete nature of the observed data (i.e. lack of information regarding individual

231 exposure/infection status). For each pathogen, the number of sub-group distributions

232 hypothesized to exist ranged from 1 to 3 , and these distributions compared by the Akaike

233 Information Criterion (AIC). The AIC adjusts the log likelihood by the number of parameters

234 involved in the model. Probabilities of correct classification or misclassification for a future

235 sample were developed for each final model. Fujii et al. (2014) applied the FMM approach to

236 identify underlying distributions of exposed and infected populations to develop surveillance of

237 tropical diseases. In addition, several underlying (mixed) distributions were considered. These

238 include the Gaussian, log normal and their mixtures, and the truncated Gaussian. Fifty-four

239 plasma samples were assayed by an independent laboratory and their resulting immune status as

240 (presumably) negative/positive compared to predictions from the respective mixed models.

241 Analysis was performed using SAS (Cary NC, version 9.3) and Microsoft Excel 13.

\section{$243 \quad 3 . \quad$ Results}

244 3.1. Experimental design

Our analysis involved an examination of a range of concentrations for each immunoassay

246 factor (Table 1). Experiments were designed using commercial animal-derived IgG antibody

247 concentrations to confirm that the antigens were properly coupled to microspheres and provided

248 a rough estimate of how the beads would react in the presence of antibodies in a human sample.

249 Table 2 provides a sample of the DOE design matrix with MFI response. A preliminary DOE

250 attempted to model the behavior of all of the antigens in one design but resulted in lack of fit due 
251 to the differences between the antigens in the assay. Hence, separate D-Optimal RSM were

252 developed and run to assess the response profile of each individual antigen. Each of the models

253 met the underlying conditions of normality, independence and non-constant variance. Thus, they

254 were sufficient for use in characterizing the impact of parameter changes and optimizing reagent

255 concentrations for each Ag. As expected, analysis of variance (ANOVA) results reflected that

256 the concentration of $1^{\circ} \mathrm{Ab}$ is the dominant factor impacting the MFI response, followed by the $2^{\circ}$

$257 \mathrm{Ab}$ concentration and the $\mathrm{BC}\left(1^{\circ} \mathrm{Ab}\right.$ and $\left.2^{\circ} \mathrm{Ab}\right)$ and $\mathrm{AC}\left(\mathrm{Ag}\right.$ and $\left.2^{\circ} \mathrm{Ab}\right)$ interactions (Fig. 1).

258 Multiple two factor interactions (2FI) were identified in the ANOVA for E. coli O157:H7. $H$.

259 pylori and C. jejuni also had a number of statistically significant higher order terms. Such

260 observations provide insight into the Ag specific variations observed in the MFI response as a

261 function of changes in reagent concentrations. These differences have a direct impact on the

262 development of the multiplex immunoassay, and by understanding and accounting for them, we

263 were able to determine the settings that worked best in the complete multiplex immunoassay.

264 Since the main goal of the DOE was to minimize the reagent concentrations necessary to

265 elicit maximum MFI response from the coupled beads, the numerical optimization tool in Design

266 Expert was employed to optimize each of the Ag specific models with the goal of maximizing

$267 \mathrm{MFI}$ while imposing equally weighted minimization constraints on $\mathrm{Ag}, 1^{\circ} \mathrm{Ab}, 2^{\circ} \mathrm{Ab}$

268 concentrations and standard error in MFI. The optimization results for the Ag specific studies

269 are summarized in Figure 2 (upper panel: RSM plots, lower panel: optimized concentrations).

270 Note, there is quite a distinctive profile for each Ag. The response surface plots developed from

271 the models reflect significant curvature in the $C$. jejuni and $H$. pylori response surfaces resulting

272 from multiple interaction and higher order effects. Such a finding indicates there are complex

273 factor effects for these organisms and the DOE for these antigens may be augmented with 
274 additional runs and center points to more accurately capture the response characteristics.

275 However, the designs used in this work provide enough information to compare and contrast key 276 factors for the multiplex immunoassay.

277 While the recommended $2^{\circ} \mathrm{Ab}$ level is similar for the Ags, the optimal $\mathrm{Ag}$ and $1^{\circ} \mathrm{Ab}$ for 278 E.coli O157:H7 and S. typhimurium tended to be much higher, yet elicited a lower MFI than for 279 H. pylori and C. jejuni. As a means of demonstrating the impact of optimizing the immunoassay 280 parameters with the goal of minimizing reagent use and maximizing MFI response, the Ag, $1^{\circ}$ $281 \mathrm{Ab}$ and $2^{\circ} \mathrm{Ab}$ concentrations from the $\mathrm{Ag}$ specific studies were compiled (Fig. 2: lower panel) 282 and compared to the mean of the reagent concentration range for the original assay design (Table 283 1). Results illustrate that while the mean amount of the $1^{\circ} \mathrm{Ab}$ concentration is nearly identical, 284 the concentrations of $\mathrm{Ag}$ and $2^{\circ} \mathrm{Ab}$ are dramatically different (Fig. 2) between the mean of DOE 285 optimized results (Table 1) and original assay. Hence, applying DOE to optimize the 286 immunoassay resulted in a recommendation of significant reductions in $\mathrm{Ag}, 1^{\circ} \mathrm{Ab}$ and $2^{\circ} \mathrm{Ab}$ 287 concentrations $(62.68 \%, 6.81 \%$ and $41.87 \%$, respectively).

\subsection{Cross-reactivity}

Cross-reactivity was used as an indicator of assay specificity and is reported in Table 3.

291 Antigens highlighted in gray were not included in the final multiplex panel because the

292 maximum MFI detected during coupling confirmation tests was less than 18,000 and/or cross-

293 reactivity between a primary detection antibody and non-target antigen was greater than $10 \%$ by

294 MFI. Among those antigens included in the assay, two exceptions were made. Cross-reactivity

295 between the two noroviruses was $84.9 \%$ when using the guinea pig anti-norovirus polyclonal

296 antibody. This was expected because the primary detection antibody was developed by 
297 immunizing guinea pigs with multiple genotypes of GI and GII noroviruses. However, when

298 rabbit anti-GII.4 primary detection antibody was employed, cross-reactivity with the GI Norwalk

299 virus antigen was reduced to $0.4 \%$. Also, $20.6 \%$ cross-reactivity was detected between the

300 hepatitis A virus antigen and anti-T. gondii antibody. This cross-reactivity was not seen with

301 further tests using human derived positive and negative control samples for HAV or T. gondii.

302 Thus, both antigens were included in the multiplex panel. All other antigens tested achieved

303 maximum reporter signals during coupling confirmation and demonstrated minimal cross-

304 reactivity.

305

\subsection{Plasma sample analysis}

307 Of the diagnostically characterized plasma samples for H. pylori, T. gondii, and HAV (n

$308=33$ ) using the optimized DOE settings, the accuracy of the Luminex assay was $87.5 \%, 100 \%$

309 and $89 \%$ respectively. One $H$. pylori characterized negative sample had an MFI value above our

310 cut-off while two HAV samples that had been characterized as positive by the vendor were

311 negative in our assay. However, because the specific antibody concentrations in the plasma

312 samples were not determined for these samples, it was not possible to conclude whether there

313 was a sensitivity issue between the testing methods employed by the vendors and the Luminex

314 assay. The S. typhimurium and L. pneumophila antigens did not detect the presence of IgG

315 antibodies in their corresponding characterized samples $(n=21)$ but antibodies to other antigens

316 were detected in those samples. The range of MFI minus the background values of three

317 antigens (H. pylori, T. gondii and hepatitis A virus) where characterized positive and negative

318 plasma samples were available are shown in Figure 3 (upper panel). The $\mathrm{S} / \mathrm{N}$ ratio of the

319 characterized positive versus the characterized negative samples was computed by dividing the 
MFI reading for each antigen in the assay by the MFI reading of the uncoupled control beads for each well. The mean $\mathrm{S} / \mathrm{N}$ of the positively characterized samples (48.13) was 4.4 times that of negative samples (10.94). All 54 characterized plasma samples were also tested for the presence of antibodies against the antigens for which they had not been previously tested. Many of the samples were positive for other antigens on the panel as indicated by the MFI (Fig. 3, lower panel).

Figure 4 graphically shows results from the finite mixed modeling of the observed mixed distribution of the respective targets in all 54 characterized plasma samples by MFI. Parameters of the underlying populations and cut-off points are shown in Table 4. The left three columns give the $\log _{10}$ mean and standard deviation of the distribution of $\log _{10}$ MFI among presumed unexposed persons, along with the $\log _{10} \mathrm{MFI}$ at the 95 th percentile for these distributions. This results in a cut-off value for classifying an individual as unexposed (or "minimally exposed") based on the $95^{\text {th }}$ percentile of their distribution, beyond which such individuals' exposure statuses would be improperly declared as positive (i.e., "false positives"). Correspondingly, the second set of three columns give $\log _{10}$ mean and standard deviation among presumed "positive" persons along with their probability of false identification as negative samples ("false negatives"). In the cases of Norwalk virus and rotavirus, where three compartment mixed models were developed to better characterize the populations, these comparisons are made among all three possible combinations of components, giving the cut-off values for false positives among presumed unexposed and unexposed populations and corresponding false negative rates among individuals in the next higher exposure category based on these cut-offs. In the case of $C$. jejuni, observed distribution of $\log _{10} \mathrm{MFI}$ is described well by a single lognormal distribution. $\log _{10}$ MFI distribution of $C$. jejuni is unimodal with a comparative high 
mean (7.8) and standard deviation (1.1). All others indicate generally excellent discrimination between exposed and unexposed subpopulations. Based on the estimates from the mixed models, Table 4 gives the implied false negative rates based on a pre-determined fixed false positive rate of 0.05 . For Norwalk virus and rotavirus, three compartment mixed models were optimal. In the case of Norwalk virus, separation between unexposed and exposed populations were clear.

However the middle "minimally exposed," population was ambiguous on the basis of its overlap with the two extreme populations ( 0.522 and 0.340 false negative rates). Rotavirus infectivity populations lack this ambiguity, with clear separations and small probabilities of false negatives regardless of true exposure status.

\section{Discussion and Conclusions}

The design of experiments (DOE) method was successfully implemented to optimize coupling confirmation reagent concentrations in the multiplex immunoassay. This approach enabled us to determine which of the antigens could be utilized in the multiplex with little to no cross-reactivity, and which ones to exclude pending further studies. Moreover, we were able to observe the differences in behavior that antigens display when used in either a monoplex or a multiplex immunoassay. Antigens from G. lamblia, Vibrio species and Shigella species were removed from the multiplex assay because they cross-reacted with antibodies against other antigens in the multiplex assay. More specific antigen-antibody pairs will have to be tested to address the cross-reactivity issues. Although coupling confirmation and cross-reactivity tests for E. coli $\mathrm{O} 157: \mathrm{H7}$, L. pneumophila and S. typhimurium were adequate, these assays would need to be further validated with either positive control samples or observed positivity in human saliva serum samples to ensure identification of samples that are positive in a population. The 
characterized samples for L. pneumophila and S. typhimurium did not produce the results expected for their corresponding antigens, therefore these antigens were removed from the multiplex immunoassay pending further studies. A key benefit of the DOE approach is the ability to clearly identify important interactions between test parameters that would have been lost using the traditional OFAT approach. Previous studies have also made this observation (Altekar et al., 2007, Joelsson et al., 2008, Ray et al., 2009).

DOE is an iterative process that affords the ability to derive valuable knowledge about a system with a minimum number of experiments (Chen et al., 2012). This was certainly the case in our study. It would have been very expensive, labor intensive, and time consuming to attempt to determine optimal concentrations for all of the antigens in our multiplex immunoassay using OFAT. While OFAT is useful for studying main effects, it involves assessing each factor separately and provides little information on how factors interact. For example, it would appear that maximizing the $\mathrm{Ag}$ or $2^{\circ} \mathrm{Ab}$ concentrations would result in greater antibody binding, however we discovered that the interaction between the $1^{\circ} \mathrm{Ab}$ and $2^{\circ} \mathrm{Ab}$ concentration $(\mathrm{BC})$ and $\mathrm{Ag}$ and $2^{\circ} \mathrm{Ab}$ concentrations (AC) were more critical than increasing the $\mathrm{Ag}$ concentration alone. In fact, we observed that increasing the Ag concentration tended to have an inverse impact on MFI response. By simply performing the random experiments as suggested by DOE which represent the range of values for each parameter, the optimal concentrations for the antigens in the assay were determined.

The mean reagent concentrations computed from the optimization results of the four monoplexes (performed separately for H. pylori, C. jejuni, E. coli O157:H7, and S. typhimurium) was used to set the optimal antigen, $1^{\circ}$ antibody, and $2^{\circ}$ antibody concentrations for the antigens in our multiplex immunoassay, with the exception of the noroviruses which had been previously 
optimized (Griffin et al., 2011). As demonstrated in the results of the positive and negative control human plasma sample for specific antigens and a $\mathrm{S} / \mathrm{N}$ ratio of 4.4 , the results showed considerable sensitivity and specificity when antibodies against them were present. Although the L. pneumophila and S. typhimurium assays did not perform as expected, we were able to test those characterized samples for exposure to other pathogens.

Immunoassay data can be difficult to interpret because the signals range from very high to very low, indicating a range of $\operatorname{IgG}$ reactivity that includes individuals that were recently infected, previously infected, previously vaccinated or have never been previously exposed. This makes it difficult to determine a cut-off between positive and negative individuals in a population, especially for acute infections. Finite mixed modeling offers an approach that exploits differences in the immune status of individuals in a population (Vyse et al., 2006, Fujii et al., 2014). Rota et al., (2008), used this approach to optimize cut-off points from measles serological survey data from an exposed population. Because the exposure or infection status of subjects in the study is not known, we estimate the underlying distributions of unexposed, newly exposed and formerly exposed populations that is suggested by the overall distribution of the reactivity from the data using FMM. An additional limitation is the small sample size used in this assay to set the cut-off points. The approach used here is a demonstration of how to set a cutoff point for antibody response but it is currently being used in this laboratory to analyze large data sets from human plasma and saliva collected from epidemiology studies.

This work explored the impact of key multiplex parameters on the output response (MFI) with the goal of determining optimal conditions for obtaining maximum MFI while minimizing reagent concentrations for various commercially available antigens. Multiple DOE analyses were performed, optimal settings were found and important interactions were studied. RSM was 
412 particularly beneficial in studying the full design space based on the parameter settings,

413 developing regression models to explore characteristics and optimizing reagent concentrations.

414 This approach proved effective at determining and distinguishing parameter importance as a

415 function of differences in antigen properties. DOE is a very promising tool that can be applied in

416 a variety of cases to provide insight on the impact of parameter changes, examining factor

417 interactions and optimizing experimental settings that result in time, resource and cost savings

418 even when dealing with quite complex problems. The establishment of cut-off points using

419 FMM allowed us to discriminate between individuals who were unexposed, exposed and

420 formerly exposed even with a limited sample size.

421 
422

423 Clarissa Curioso was supported through an appointment to the Research Participation Program at

424 the U.S. Environmental Protection Agency administered by the Oak Ridge Institute for Science

425 and Education through an interagency agreement between the U.S. Department of Energy and

426 U.S. EPA.

427

428

429

430

431

432

433

434

435

436

437

438

439

440

441

442

443

444
Thanks to Dr. Alec Sutherland, Arizona State University (AZ, USA) for kindly providing rabbit anti-GII.4 antibodies, Dr. Richard Ward (Children's Hospital, Cincinnati, OH) for the rotavirus VP6 antigen and Dr. Tim Wade, National Health and Environmental Effects Laboratory (USEPA, RTP, NC) for his guidance regarding finite mixed modeling.

Mr. Jatin H. Mistry, Public Health Fellow with the Association of Schools of Public Health, assisted the principal investigator (Dr. Augustine) in establishing a team of ORD scientists who served as the project's Steering Committee along with providing assistance in researching a variety of analytical platforms at the outset of the project.

The authors are grateful to Dr. Jay Garland for his critical review of the manuscript. 


\section{NOTICE}

446 The United States Environmental Protection Agency through its Office of Research and

447 Development funded and managed the research described here. It has been subjected to Agency's

448 administrative review and approved for publication. Mention of trade names or commercial

449 products does not constitute endorsement or recommendation for use.

450

451

452

453

454

455

456

457

458

459

460

461

462

463

464

465

466 
468

469

470

471

472

473

474

475

476

477

478

479

480

481

482

483

484

485

486

487

488

489

490

491

492

493

494

495

496

497

498

499

500

501

502

503

504

505

506

507

508

509

510

Altekar, M., Homon, C. A., Mohammed, A. K., Mason, S. W., et al. 2007. "Assay Optimization: A Statistical Design of Experiments Approach." Clinics in Laboratory Medicine no. 27 (1):139-154. doi: http://dx.doi.org/10.1016/j.cll.2007.01.001.

Baughman, A. L., Bisgard, K. M., Edwards, K. M., Guris, D., et al. 2004. Establishment of diagnostic cutoff points for levels of serum antibodies to pertussis toxin, filamentous hemagglutinin, and fimbriae in adolescents and adults in the United States. Clinical and diagnostic laboratory immunology, 11(6), 1045-1053.

Chen, X. C., Lei, Z, Gupta, S. and Civoli, F. 2012. "Implementation of design of experiments (DOE) in the development and validation of a cell-based bioassay for the detection of anti-drug neutralizing antibodies in human serum." Journal of Immunological Methods no. 376 (1-2):32-45. doi: http://dx.doi.org/10.1016/j.jim.2011.11.004.

Choi, K., Ng, A. H. C., Fobel, R., Chang-Yen, D. A., et al., A. R. 2013. "Automated Digital Microfluidic Platform for Magnetic-Particle-Based Immunoassays with Optimization by Design of Experiments." Analytical Chemistry. doi: 10.1021/ac401847x.

Ellington, A. A., Kullo, I. J., Bailey, K. R. and Klee, G. G. 2010. "Antibody-based protein multiplex platforms: technical and operational challenges." Clin Chem no. 56 (2):186-93. doi: 10.1373/clinchem.2009.127514.

Fu, Q., Zhu, J. and Van Eyk, J. E. 2010. "Comparison of multiplex immunoassay platforms." Clin Chem no. 56 (2):314-8. doi: 10.1373/clinchem.2009.135087.

Fujii, Y., Kaneko, S., Nzou, S. M., Mwau, M., Njenga, S. M., et al. 2014. "Serological surviellance development for tropical infectious diseases using simultaneous microsphere-based multiplex assays and finite mixed models." PLoS Negl Trop Dis no 8(7): e3040. doi: 10.1371/journal.pntd.0003040

Griffin, S. M., Chen, I. M., Fout, G. S., Wade, T. J., and Egorov, A. I. 2011. "Development of a multiplex microsphere immunoassay for the quantitation of salivary antibody responses to selected waterborne pathogens." J Immunol Methods no. 364 (1-2):83-93. doi: 10.1016/j.jim.2010.11.005.

Joelsson, D., P. Moravec, Troutman, M., Pigeon, J., and DePhillips, P. 2008. "Optimizing ELISAs for precision and robustness using laboratory automation and statistical design of experiments." J Immunol Methods no. 337 (1):35-41. doi: 10.1016/j.jim.2008.05.012.

Joyce, A. P., and Leung, S. S. 2013. "Use of response surface methods and path of steepest ascent to optimize ligand-binding assay sensitivity." Journal of Immunological Methods no. 392 (1-2):12-23. doi: http://dx.doi.org/10.1016/j.jim.2013.02.019.

Khuri, A. I. and Mukhopadhyay, S. 2010. "Response surface methodology." Wiley Interdisciplinary Reviews: Computational Statistics no. 2 (2):128-149. doi: 10.1002/wics.73.

Lilienfeld, A. M. 1983. "Practical limitations of epidemiologic methods." Environ Health Perspect no. 52:3-8.

Montgomery, D.C. 1997. Design and analysis of experiments: Wiley.

McLachlan G., and Peel, D. 2000. "Finite Mixture Models". John Wiley and Sons Inc., New York.

Ray, C. A., Patel, V., Shih, J., Macaraeg, C., et al. 2009. "Application of multi-factorial design of experiments to successfully optimize immunoassays for robust measurements of 
therapeutic proteins." J Pharm Biomed Anal no. 49 (2):311-8. doi: 10.1016/j.jpba.2008.11.039.

Rota, C. M., Massari, M., Gabutti, G., Guido, M., et al. 2008. "Measles serological survey in the Italian population: Interpretation of results using mixture model." Vaccine no. 26. 44034409. doi: 10.1016/j.vaccine.2008.05.094.

Sittampalam, G. S., Smith, W. C., Miyakawa, T. W., Smith, D. R., and McMorris, C. 1996. "Application of experimental design techniques to optimize a competitive ELISA." Journal of Immunological Methods no. 190 (2):151-161. doi: http://dx.doi.org/10.1016/0022-1759(95)00262-6.

Titterington, D. M., Smith, A. F. M., and Markov, U. E. 1985. "Statistical Analysis of Finite Mixture Distributions." New York: John Wiley \& Sons.

Vyse, A. J., Gay, N. J., Hesketh, L. M., Pebody, R., et al. 2006. "Interpreting serological surveys using mixture models: the seroepidemiology of measles, mumps and rubella in England and Wales at the beginning of the $21^{\text {st }}$ century." Epidemiol. Infect. no. 134. 1303-1312. doi: $10.1017 /$ S0950268806006340.

Xie, J., Zorman, J., Indrawati, L., Horton, M., et al. 2013. "Development and optimization of a novel assay to measure neutralizing antibodies against Clostridium difficile toxins." Clin Vaccine Immunol no. 20 (4):517-25. doi: 10.1128/cvi.00549-12.

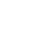

(1)




\section{$543 \quad$ Figure Legends}

544 Figure 1. Summary of the ANOVA results from the Ag specific DOE studies: The figure

545 displays the frequency of identifying certain statistically significant main and two factor

546 interaction (2FI) effects. The $1^{\circ} \mathrm{Ab}$ concentration was identified as an important factor in each

547 of the models, followed by the $2^{\circ} \mathrm{Ab}$ concentration, $\mathrm{BC}$ and $\mathrm{AC}$ interactions. Given the multiple

548 statistically significant main and 2FI identified, E. coli appears to exhibit the greatest amount of

549 complexity. Note: Factors are A: Antigen concentration, B: $1^{\circ} \mathrm{Ab}$ concentration and C: $2^{\circ} \mathrm{Ab}$

550 concentration.

552 Figure 2. Summary of DOE optimization results from Ag specific studies. The surface plots

553 present a profile of the $1^{\circ}$ and $2^{\circ} \mathrm{Ab}$. interaction and corresponding MFI response. The reagent

554 concentrations provided were determined by optimizing the response surface model given the

555 goal of minimizing reagent use while maximizing MFI response.

556

557 Figure 3. Range of Median Fluorescence Intensity minus background values for antigens in the

558 multiplex assay exposed to the 54 characterized plasma samples. Top panel: Observed MFI's for

559 the specific antigens to the characterized samples. Bottom panel: Observed MFI's to non-

560 characterized antigens in the characterized samples.

562 Figure 4. Finite mixed models for pathogens based on observed distributions. The dotted line 563 (sometimes obscured by the component distributions, which are differentiated by solid and 
564 dashed lines) is the sum of the component distributions, equal to 1. Relative areas under the

565 component curves give the probability of false positive to false negative (from left to right). For

566 comparison, histograms of the observed distribution are shown by bars and percent of the sample

567 falling with each range are indicated by the values on the $y$-axis. Cutoff values, defining the $95^{\text {th }}$

568 percentile of the distribution of $\log _{10}$ MFI among the presumed "unexposed" individuals is

569 shown as a vertical line.

571 Table 1. Factor and levels for the designed experiments. These settings were developed from

572 preliminary experiments which indicated that reagent concentrations varied from organism to

573 organism; hence we explored a range of concentrations.

575 Table 2. Sample of the DOE design matrix with corresponding MFI response.

577 Table 3. Evaluation of multiplex assay specificity. Maximum signal observed between target 578 antigen and detection antibody as well as percent cross-reactivity of signal obtained between non 579 target antigen and detection antibody are presented. Shaded (gray) area indicates antigens not 580 included in final assay.

582 Table 4. Cut-offs and implied false negative rates based on calculated means and standard 583 deviation of underlying unexposed and exposed populations. 


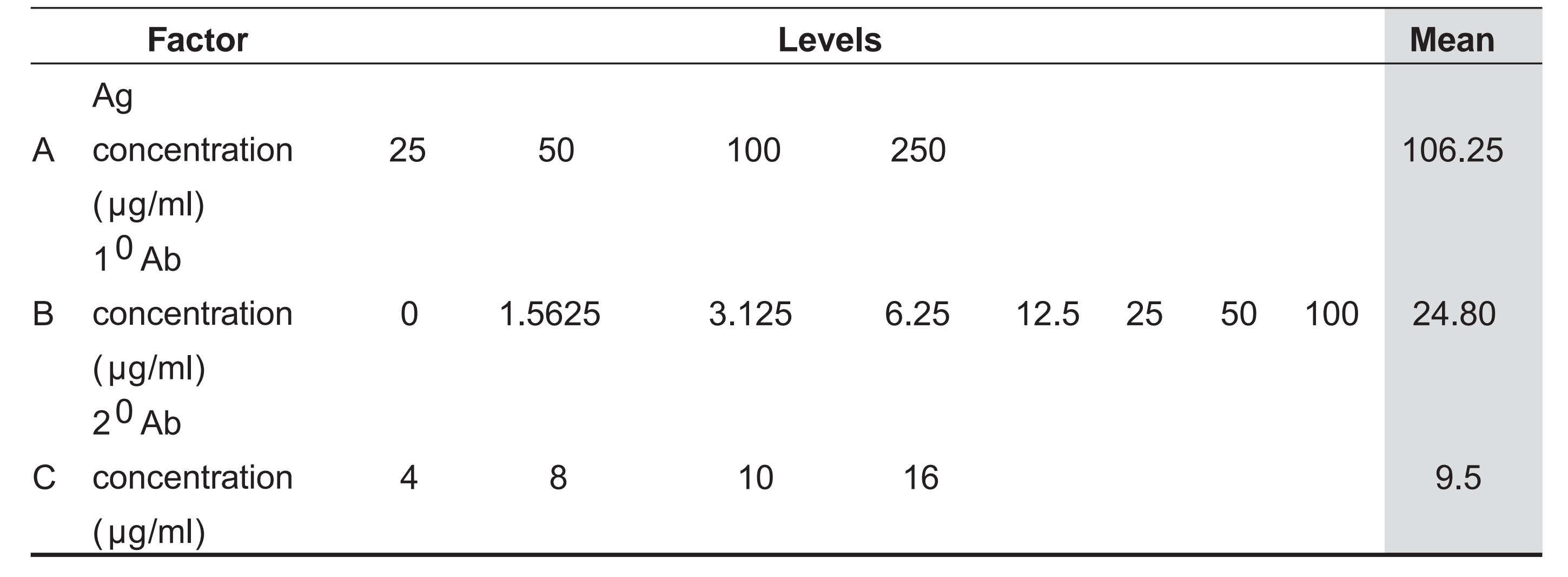




\begin{tabular}{|cccccc|}
\hline & & A & B & C & \\
\cline { 3 - 6 } Run & Ag name & $\begin{array}{c}\mathrm{Ag} \\
\text { concentration } \\
(\mu \mathrm{g} / \mathrm{ml})\end{array}$ & $\begin{array}{c}1^{0} \mathrm{Ab} \\
\text { concentration } \\
(\mu \mathrm{g} / \mathrm{ml})\end{array}$ & $\begin{array}{c}2^{0} \mathrm{Ab} \\
\text { concentration } \\
(\mu \mathrm{g} / \mathrm{ml})\end{array}$ & MFI \\
\hline 1 & H. pylori & 25 & 100 & 8 & 23722 \\
2 & H. pylori & 250 & 50 & 4 & 18933 \\
3 & C. jejuni & 250 & 3.125 & 4 & 18558.5 \\
4 & E. coli 0157:H7 & 50 & 50 & 10 & 3500 \\
5 & S. typhimurium & 25 & 3.125 & 16 & 977.5 \\
6 & H. pylori & 250 & 6.25 & 10 & 17971.5 \\
7 & E. coli 0157:H7 & 50 & 3.125 & 4 & 2094 \\
8 & E. coli 0157:H7 & 250 & 50 & 10 & 5362 \\
\hline
\end{tabular}




\begin{tabular}{|c|c|c|c|c|c|c|c|c|c|c|c|c|c|}
\hline Target analyte (catalog no.) & 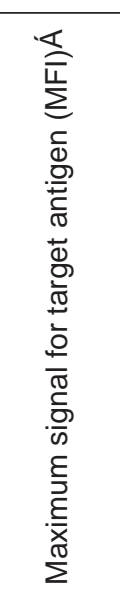 & 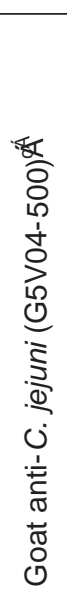 & 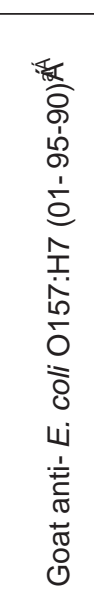 & 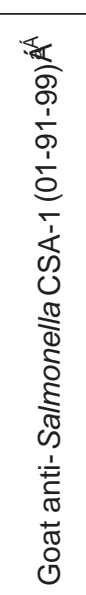 & 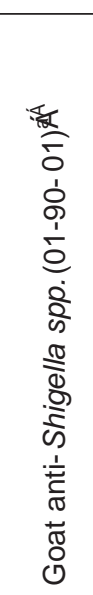 & 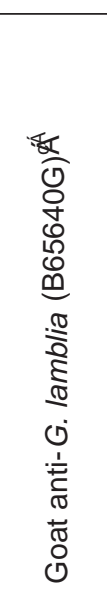 & 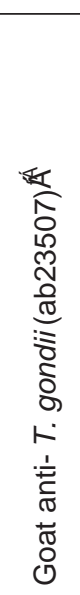 & 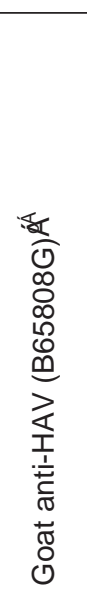 & 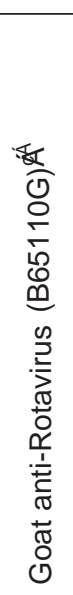 & 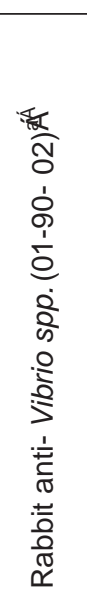 & 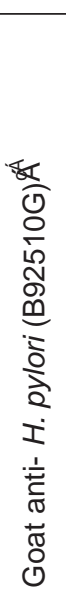 & 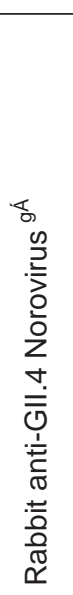 & 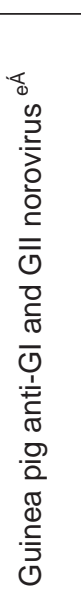 \\
\hline C. jejuni intact cells $(50-92-93)^{a}$ & 25820 & & 2.5 & 7.8 & 18.2 & 109.7 & 3.5 & 6.7 & 0.3 & 22.8 & 3.8 & 3.3 & 0.2 \\
\hline E. coli $\mathrm{O} 157: \mathrm{H} 7$ intact cells $(50-74-01)^{\mathrm{a}}$ & 20550 & 1.6 & & 27.9 & 9.8 & 58.6 & 3 & 1.5 & 0.3 & 7.9 & 0.3 & 1 & 0.2 \\
\hline S. typhiintact cells $(50-74-01)^{a}$ & 20688 & 1.4 & 4.2 & & 18.7 & 86.1 & 2.1 & 4 & 0.4 & 13.5 & 0.4 & 2.6 & 0.3 \\
\hline Shigella spp.intact cells $(50-90-01)^{a}$ & 17258 & 3.9 & 12.3 & 37.6 & & 119.1 & 2.3 & 10.4 & 0.9 & 94.2 & 1.4 & 3.6 & 0.8 \\
\hline G. lamblia lysates ${ }^{\text {b }}$ & 5545 & 0.7 & 1.4 & 2 & 1.3 & & 3.2 & 0.8 & 0.1 & 0.9 & 0.1 & 0.1 & 0.02 \\
\hline T. gondii p30 (R86300) ${ }^{\mathrm{C}}$ & 26199 & 0.9 & 2 & 2.1 & 3.7 & 138.4 & & 0.6 & 0.1 & 2.6 & 0.1 & 1.4 & 0.03 \\
\hline HAV from cell culture lysates $(8505)^{\mathrm{c}}$ & 18207 & 4.4 & 16.1 & 17 & 2 & 179.4 & 20.6 & & 2.7 & 1.5 & 0.3 & 4.6 & 0.1 \\
\hline Rotavirus VP6 ${ }^{d}$ & 26114 & 1.1 & 2.6 & 3 & 2.3 & 107.1 & 5.1 & 4 & & 1.2 & 0.3 & 1 & 0.1 \\
\hline Vibrio spp. intact cells (50-90-02) ${ }^{\mathrm{a}}$ & 19565 & 3.7 & 12.9 & 33.1 & 26.2 & 170.2 & 6.3 & 6.5 & 1.5 & & 1.6 & 5.3 & 1.3 \\
\hline H. pylori lysate $(\mathrm{R} 14101)^{\mathrm{c}}$ & 19197 & 1.7 & 1.6 & 2.1 & 1.5 & 60.9 & 4.8 & 2.1 & 0.5 & 1 & & 5.1 & 0.4 \\
\hline Norovirus VA387 P particle ${ }^{\mathrm{e}}$ & 25912 & 1.6 & 3 & 4.7 & 4.3 & 96.3 & 2.1 & 1.2 & 0.2 & 3.9 & 0.3 & & 84.9 \\
\hline Norwalk virus $\mathrm{P}$ particle ${ }^{\mathrm{e}}$ & 28605 & 2 & 3.6 & 8.3 & 5.6 & 134.8 & 6.1 & 2.4 & 0.3 & 5.1 & 0.6 & 0.4 & \\
\hline BSA control & ND & 0.8 & 2 & 1.5 & 1.8 & 88.6 & 1.5 & 0.5 & 0.1 & 0.9 & 0.1 & 0.1 & 0.03 \\
\hline
\end{tabular}


exposed" samples

"Positive" samples

\begin{tabular}{|c|c|c|c|c|c|c|}
\hline & Mean & SD & Cutoff $^{1}$ & Mean & SD & $P(F)^{2}$ \\
\hline Campylobacter & 3.370 & 0.4934 & - & \multicolumn{3}{|c|}{$\left(1\right.$ Component Model) ${ }^{3}$} \\
\hline Toxoplasma & 2.381 & 0.3061 & 2.884 & 3.614 & 0.2242 & 0.001 \\
\hline Helicobacter & 2.546 & 0.1425 & 2.781 & 3.735 & 0.4896 & 0.026 \\
\hline Hepatitis A & 2.615 & 0.4031 & 3.279 & 3.872 & 0.3004 & 0.017 \\
\hline NoroVirus VA387 & 3.007 & 0.2120 & 3.356 & 4.028 & 0.4309 & 0.059 \\
\hline \multicolumn{7}{|l|}{ Norwalk } \\
\hline - Component 1 vs. 2 & 3.373 & 0.4482 & 4.111 & 4.104 & 0.1910 & 0.522 \\
\hline - Component 1 vs. 3 & $"$ & $"$ & $"$ & 2.324 & 0.0091 & $<0.001$ \\
\hline - Component 2 vs. 3 & 4.104 & 0.1910 & 4.419 & $"$ & $"$ & 0.340 \\
\hline \multicolumn{7}{|l|}{ Rotavirus } \\
\hline - Component 1 vs. 2 & 3.549 & 0.1824 & 3.849 & 4.126 & 0.1334 & 0.019 \\
\hline - Component 1 vs. 3 & $"$ & $"$ & $"$ & 4.415 & 0.0382 & $<0.001$ \\
\hline - Component 2 vs. 3 & 4.126 & 0.1334 & 4.346 & $"$ & $"$ & $<0.001$ \\
\hline
\end{tabular}

${ }^{1}$ Cutoff is determined as that reactivity (log10MFI) at the 95th percentile of the distribution of $\log _{10} \mathrm{MFI}$ values among unexposed/minimally exposed subjects, equivalent to a $5 \%$ false positive rate. ${ }^{2} \mathrm{P}(\mathrm{F}-)$ is calculated from the distribution of positive (i.e. exposed) subjects as the proportion falsely categorized unexposed/minimally exposed based on the cutoff value. ${ }^{3}$ Observed $\log _{10} \mathrm{MFI}$ among Campylobacter antibody samples are best modeled by just a single distribution. This describes a wide range of exposures, as evidenced by the large log standard deviation. 
Figure 1

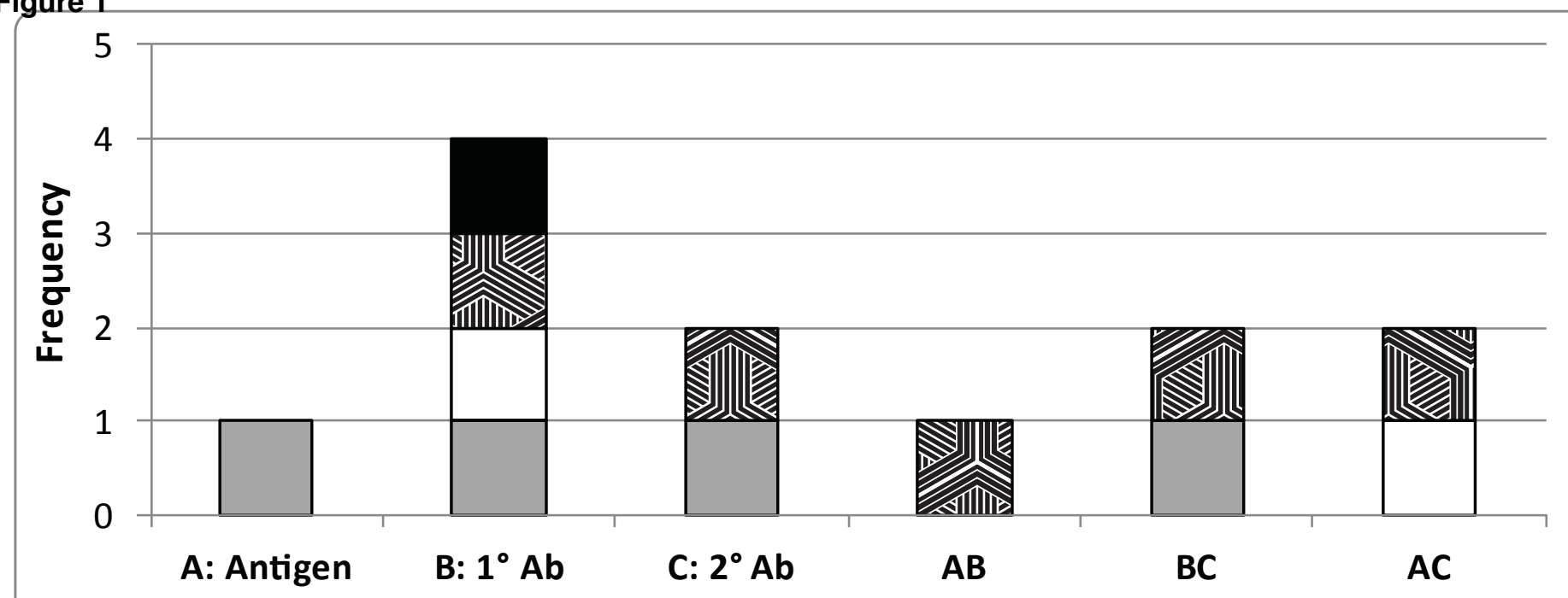

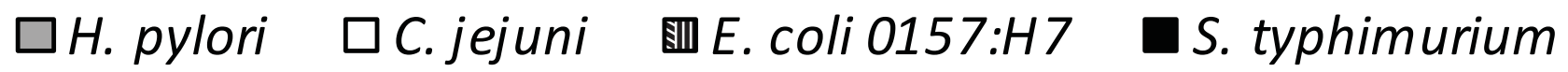

p-Values from Separate RSM ANOVAs: Main and 2FI

\begin{tabular}{|rcccccc|}
\hline Antigen & A & B & C & AB & BC & AC \\
\hline H. pylori & 0.0114 & $<0.0001$ & 0.0064 & & 0.0416 & \\
C. jejuni & & $<0.0001$ & & & & 0.0246 \\
E. coli0157:H7 & & $<0.0001$ & 0.0002 & 0.0029 & 0.0173 & 0.0088 \\
S. typhimurium & & 0.0019 & & & & \\
\hline
\end{tabular}



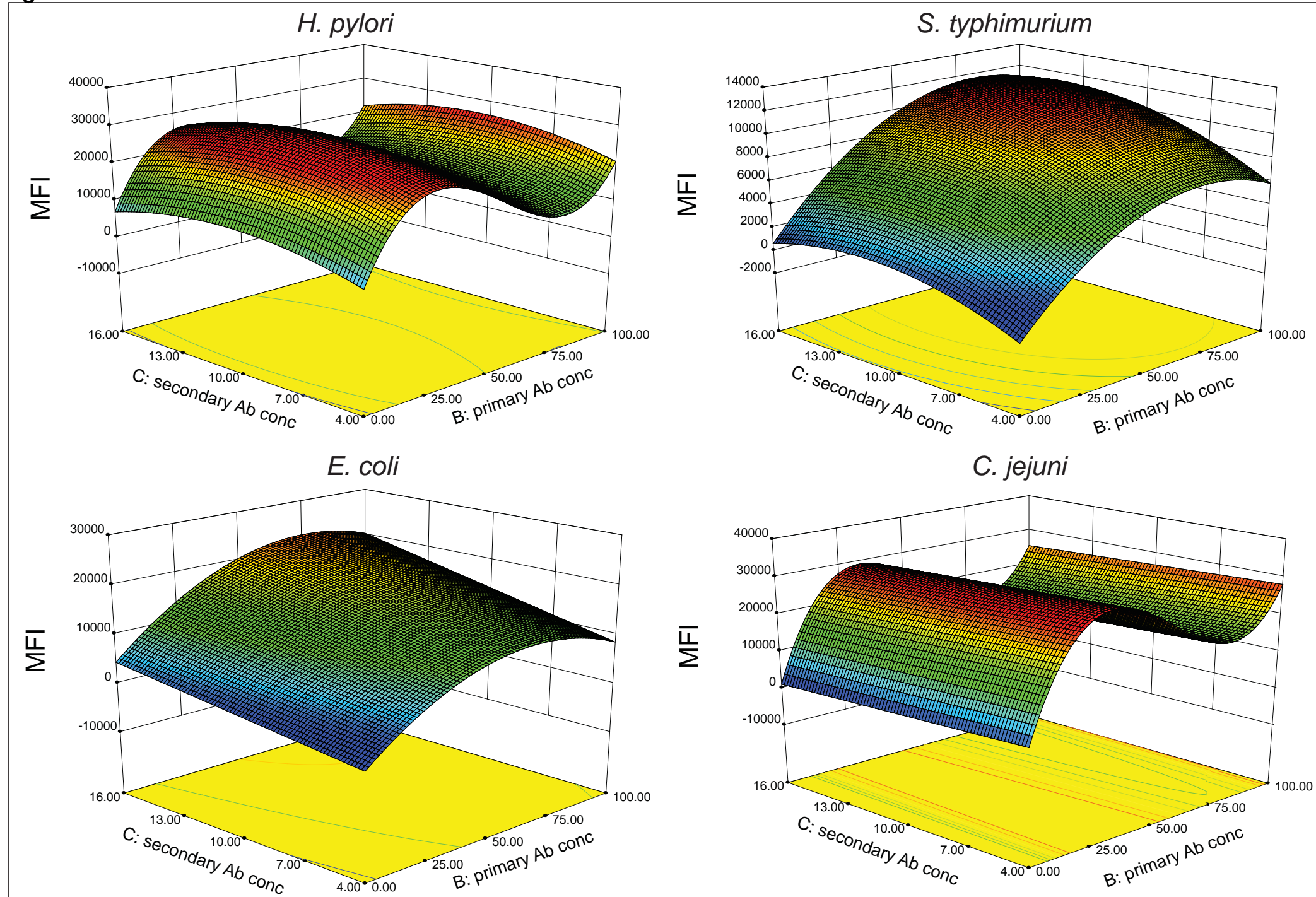

\begin{tabular}{|c|c|c|c|c|}
\hline \multicolumn{4}{|c|}{ Optimized Concentration ( $\mu \mathrm{g} / \mathrm{ml})$} & \multirow[b]{2}{*}{ MFI } \\
\hline Antigen & Antigen & $1^{0} \mathrm{Ab}$ & $2^{0} \mathrm{Ab}$ & \\
\hline H. pylori & 29.89 & 13.45 & 5.70 & 23071.90 \\
\hline C. jejuni & 36.20 & 12.80 & 4.42 & 23437.50 \\
\hline E. coli $0157: \mathrm{H} 7$ & 48.45 & 28.48 & 6.43 & 9654.91 \\
\hline S. typhimurium & 44.08 & 37.73 & 5.54 & 6668.49 \\
\hline Mean & 39.66 & 23.12 & 5.52 & \\
\hline
\end{tabular}


Figure 4
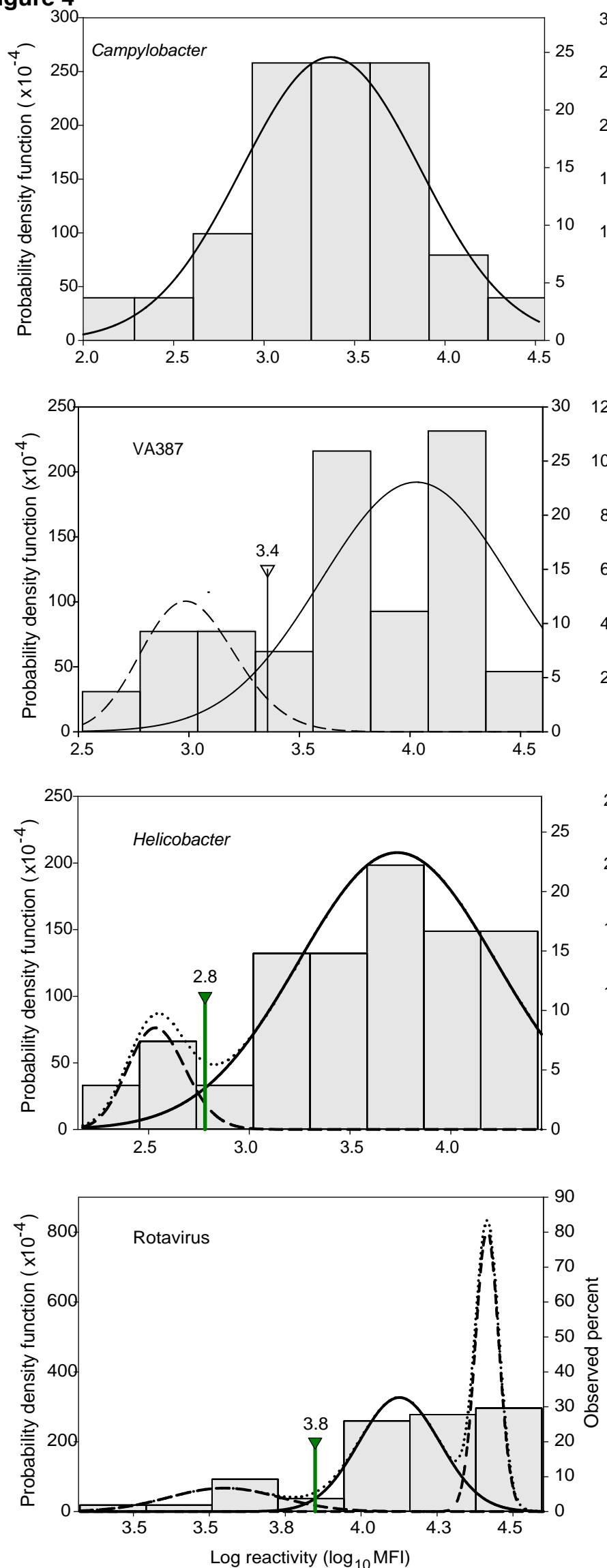

Log reactivity $\left(\log _{10} \mathrm{MFI}\right)$
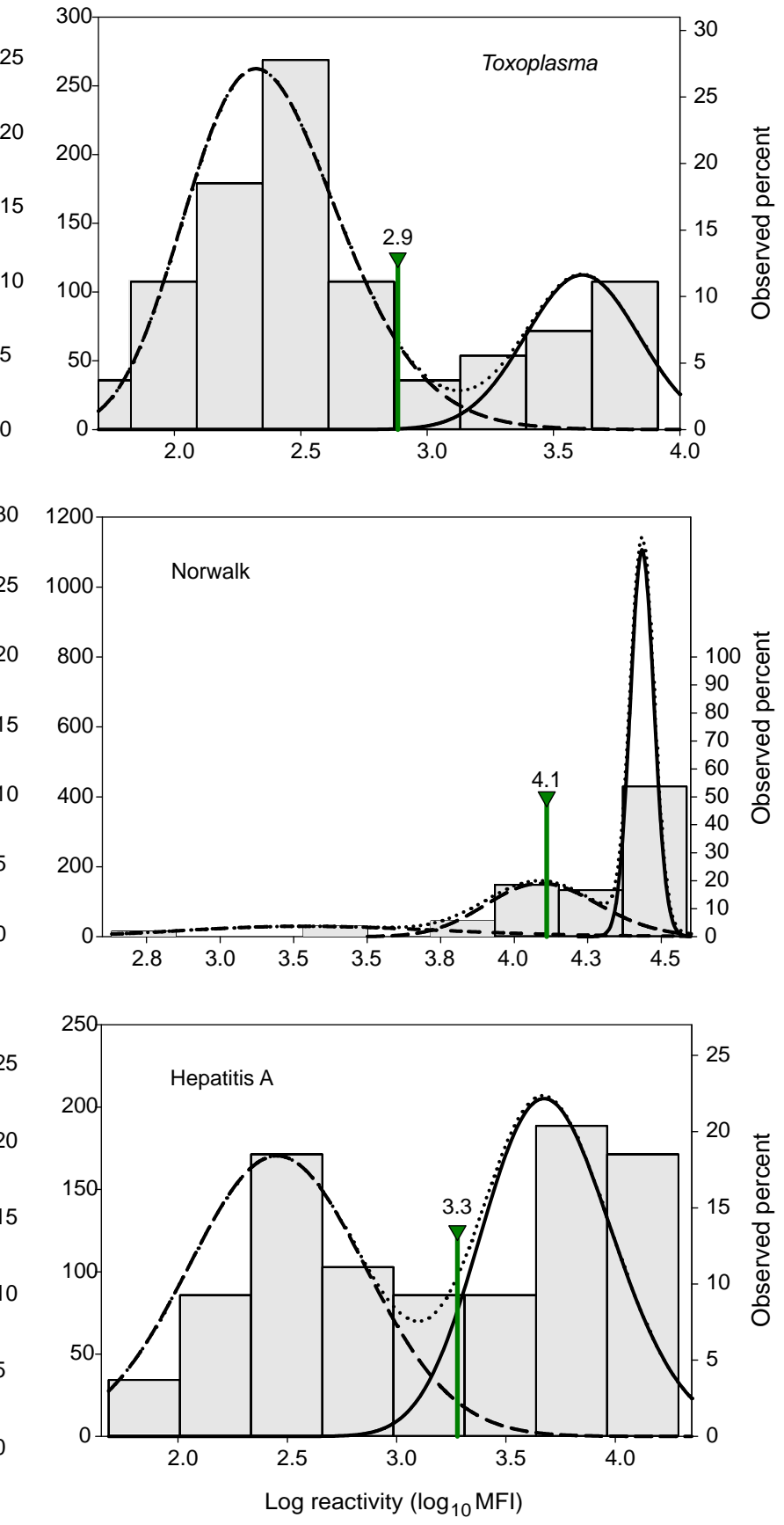